\title{
ZEROS OF A ONE-PARAMETER FAMILY OF HARMONIC TRINOMIALS
}

\author{
MICHAEL BRILLESLYPER, JENNIFER BROOKS, MICHAEL DORFF, \\ RUSSELL HOWELL, AND LISBETH SCHAUBROECK
}

(Communicated by Jeremy Tyson)

\begin{abstract}
It is well known that complex harmonic polynomials of degree $n$ may have more than $n$ zeros. In this paper, we examine a one-parameter family of harmonic trinomials and determine how the number of zeros depends on the parameter. Our proof heavily utilizes the Argument Principle for Harmonic Functions and involves finding the winding numbers about the origin for a family of hypocycloids.
\end{abstract}

\section{INTRODUCTION}

Let $f=u+i v$ be a continuous complex-valued harmonic function, where both $u$ and $v$ are real-valued harmonic functions. Such a function can be expressed as $f=h+\bar{g}$, where $h$ and $g$ are analytic.

A familiar fact about analytic functions is that they are sense-preserving (or orientation-preserving) at all points at which the derivative is not zero. Complexvalued harmonic functions, however, may be sense-preserving in one region and sense-reversing in another. Lewy's Theorem [13] implies that $f=h+\bar{g}$ is locally univalent and sense-preserving if and only if $h^{\prime}(z) \neq 0$ and the dilatation function $\omega$ of $f$, defined by $\omega(z)=g^{\prime}(z) / h^{\prime}(z)$, satisfies $|\omega(z)|<1$. The dilatation function $\omega(z)$ can be viewed as a measure of how far $f(z)$ is from being analytic; $\omega(z) \equiv 0$ for analytic functions, and $|\omega(z)| \leq k<1$ is the well-studied class of quasiconformal functions [1].

Much work has been done examining the similarities and differences between analytic and harmonic functions. Many familiar results for analytic functions hold for complex-valued harmonic functions with only slight modifications. The harmonic analog of the Argument Principle for Analytic Functions is a case in point [7].

Argument Principle for Harmonic Functions: Let $D$ be a Jordan domain with boundary $C$. Suppose that $f$ is harmonic on $D$, continuous on $\bar{D}, f \neq 0$ on $C$, and there are no zeros $z_{0}$ of $f$ in $D$ for which $\left|\omega\left(z_{0}\right)\right|=1$. Then the total change in the argument of $f(z)$ as $C$ is traversed in the positive direction is $2 \pi N$, where $N$ is the number of zeros of $f(z)$ in $D$ counted according to their multiplicity.

Received by the editors December 16, 2019, and, in revised form, May 15, 2020.

2010 Mathematics Subject Classification. Primary 30C15. 
The multiplicity of a zero $z_{0}$ of a complex-valued harmonic function is defined via its power series expansion about the zero. That is, let

$$
f(z)=h(z)+\overline{g(z)}=a_{0}+\sum_{j=r}^{\infty} a_{j}\left(z-z_{0}\right)^{j}+\overline{\left(b_{0}+\sum_{j=s}^{\infty} b_{j}\left(z-z_{0}\right)^{j}\right)},
$$

where $a_{r} \neq 0$ and $b_{s} \neq 0$. If $z_{0}$ is in the sense-preserving region of the plane, then $r \geq s$ and the order of the zero is $r$. If $z_{0}$ is in the sense-reversing region of the plane, then $s \geq r$ and the order of the zero is defined to be $-s$. For more information about complex-valued harmonic functions, see [4], [5], and [6].

A tantalizing problem is to determine the number of zeros of a complex-valued harmonic polynomial. For analytic polynomials, the Fundamental Theorem of Algebra stipulates that the number of zeros counting multiplicity equals the degree of the polynomial. In the harmonic case, the results are not so straightforward. If $p=h+\bar{g}$ is a harmonic polynomial for which the degree of $h$ is $n$, the degree of $g$ is $m$, and $m \leq n$, then Sheil-Small [17] conjectured that the maximum number of zeros of $p$ is $n^{2}$. Wilmshurst [18] proved this conjecture and provided an example to show that $n^{2}$ is sharp. Independently, Peretz and Schmid [16] also proved the conjecture, while Bshouty et al. [3] provided another example to establish the sharpness of the bound. Wilmshurst further conjectured that in the more restrictive case in which $1 \leq m \leq n-1, p$ has at most $m(m-1)+3 n-2$ zeros. Khavinson and Swiatek [9] looked at the subclass of harmonic polynomials $p(z)=h(z)-\bar{z}$ related to gravitational lensing. They showed that if the degree of $h$ is $n>1$, then the number of zeros is bounded by $3 n-2$, which satisfies Wilmshurst's conjecture. However, Lee et al. [12 later showed that Wilmshurst's conjecture is not true in general.

Recently, Melman [14] investigated trinomials of the form $q(z)=z^{n}-a z^{k}-1$, where $1 \leq k \leq n-1, n \geq 3, a \in \mathbb{C}$, and $\operatorname{gcd}(n, k)=1$. He gleaned information relating to the location of the zeros of $q$. In a similar fashion, Brilleslyper and Schaubroeck [1, 2], considered the family of trinomials $p(z)=z^{n}+z^{k}-1$, where $1 \leq k \leq n-1, n \geq 2$, and derived a formula for the number of zeros of $p$ located on the unit circle. Howell and Kyle 8] then proved a conjecture in [2] to determine the number of zeros of this same trinomial $p$ in the interior and exterior of the unit circle. In this paper, we look at a harmonic equivalent of these trinomials. That is, we consider the family of harmonic trinomials

$$
p_{c}(z)=z^{n}+c \bar{z}^{k}-1
$$

where $1 \leq k \leq n-1, n \geq 3, c \in \mathbb{R}^{+}$, and $\operatorname{gcd}(n, k)=1$. We are interested in how the number of zeros of $p_{c}(z)$ changes as $c$ varies. Our main theorem is the following.

Theorem 1.1. Let $p_{c}(z)$ be as in (1.2) and let $N=\lfloor k / 2\rfloor+1$. There exist $N$ critical values $c_{j}$, with $0<c_{1}<c_{2}<\cdots<c_{N}$, such that

(a) if $0 \leq c<c_{1}$, then $p_{c}(z)$ has $n$ distinct zeros,

(b) if $c_{j}<c<c_{j+1}$ for some $1 \leq j \leq N-1$, then $p_{c}(z)$ has $n+4 j-2$ distinct zeros, and

(c) if $c>c_{N}$, then $p_{c}(z)$ has $n+2 k$ distinct zeros.

We will prove this theorem in the following section. In the process we also show that $c_{1}=\left(\frac{n}{n+k}\right)\left(\frac{n+k}{k}\right)^{k / n}>1$ and $c_{N} \leq\left(\frac{n}{n-k}\right)\left(\frac{n-k}{k}\right)^{k / n}$. The following example and accompanying figure provide an illustration. 
Example 1.2. Consider $p_{c}(z)=z^{5}+c \bar{z}^{3}-1$, so that, with the notation of Theorem 1.1. $N=2, c_{1}=\left(\frac{5}{8}\right)\left(\frac{8}{3}\right)^{3 / 5} \approx 1.126$, and $c_{2}<\left(\frac{5}{2}\right)\left(\frac{2}{3}\right)^{3 / 5} \approx 1.960$. As Figure 1 illustrates, when $c=1<c_{1}$, the trinomial $p_{c}(z)$ has $n=5$ zeros. When $c=1.5$, corresponding to the case $c_{1}<c<c_{2}, p_{c}(z)$ has $5+4(1)-2=7$ zeros. Finally, when $c=3>c_{2}$, the number of zeros has increased to $5+2(3)=11$.

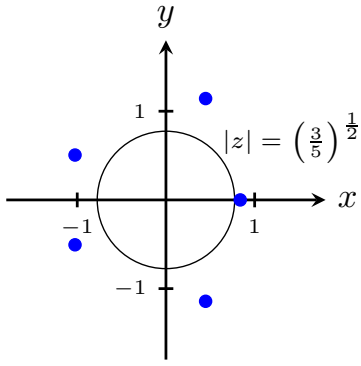

(A) Zeros of $z^{5}+\bar{z}^{3}-1$

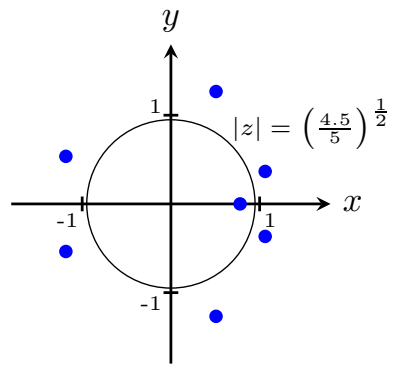

(B) Zeros of $z^{5}+1.5 \bar{z}^{3}-1$

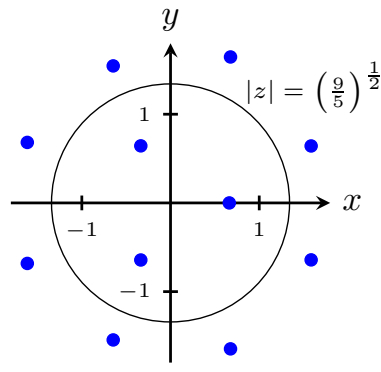

(C) Zeros of $z^{5}+3 \bar{z}^{3}-1$

Figure 1. Zeros of $p_{c}(z)$ for $c=1,1.5,3$, and circles $|z|=\left(\frac{c k}{n}\right)^{\frac{1}{n-k}}$

Theorem 1.1 states that the behavior seen for $p_{c}(z)=z^{5}+c \bar{z}^{3}-1$ is typical: the number of zeros of $p_{c}(z)=z^{n}+c \bar{z}^{k}-1$ increases from $n$ to $n+2 k$ as $c$ increases. The $c$-values at which the number of zeros increases will be called the critical c-values.

The curve in the plane that separates the sense-preserving and sense-reversing regions for $p_{c}(z)$ plays a significant role in our analysis. A straightforward computation gives that $|\omega(z)|=1$ if and only if $|z|=\left(\frac{c k}{n}\right)^{\frac{1}{n-k}}$, with $p_{c}(z)$ being sense-reversing on the interior of this circle and sense-preserving on its exterior. Because of its central role, we make the following definition.

Definition 1.3. The critical circle is $\Gamma_{c}=\left\{z:|z|=R_{c}=\left(\frac{c k}{n}\right)^{\frac{1}{n-k}}\right\}$.

Note that in Figure $1(\mathrm{~A})$ there are 5 zeros outside of $R_{c}$, in Figure $1(\mathrm{~B})$ there are 6 zeros outside of $R_{c}$ and 1 zero inside, and in Figure $1(\mathrm{C})$ there are 8 zeros outside of $R_{c}$ and 3 zeros inside. Recall from the Argument Principle for Harmonic Functions that a zero in a sense-preserving region has a positive order while a zero in a sense-reversing region has a negative order. So, in each case in Figure 1, the sum of the orders of the zeros is 5 , which is the value of $n$ for $p_{c}(z)$. In this paper we prove that transitions between numbers of zeros occur when $p_{c}\left(\Gamma_{c}\right)$ passes through the origin. Furthermore, we show that, for $0 \leq c<c_{1}$, the trinomial $p_{c}(z)$ has no zeros inside $\Gamma_{c}$, whereas for $c>c_{N}$, it has $k$ zeros inside $\Gamma_{c}$.

\section{Proof of Theorem 1.1}

To prove Theorem 1.1 we make use of a series of lemmas. First, keeping in mind that the zeros of $p_{c}(z)$ may include zeros of both positive and negative order, Lemma 2.1 concludes that, for all $c$, the sum of the orders of the zeros of $p_{c}(z)$ equals $n$. Lemma 2.2 then provides a short proof that every zero of $p_{c}(z)$ has order 1 or -1 . It is also necessary to find the winding number of the image $p_{c}\left(\Gamma_{c}\right)$ of the critical circle about the origin, because this information relates to the number of 
zeros in the sense-reversing region. Towards that end, Lemma 2.4 shows that the image $p_{c}\left(\Gamma_{c}\right)$ is a certain type of hypocycloid that is centered at -1 and whose size is affected only by $c$. Further, the geometry of the hypocycloid reveals that the number of zeros can only change with additional zeros being balanced inside and outside the critical circle $\Gamma_{c}$. Finally, Lemma 2.5 shows that $p_{c}\left(\Gamma_{c}\right)$ has $\lfloor k / 2\rfloor+1$ distinct intersections with the real axis to the right of its center at $z=-1$, and hence counts the winding number of $p_{c}\left(\Gamma_{c}\right)$ around the origin for the various values of $c$. The proof of our theorem follows by combining these lemmas.

Lemma 2.1. Let $p_{c}(z)$ be as in (1.2). For $R$ sufficiently large, the winding number of the image of $|z|=R$ under $p_{c}(z)$ around the origin is $n$. Thus, for all $c$, the sum of the orders of the zeros of $p_{c}(z)$ is $n$.

Proof. The proof follows immediately from a standard Rouché-type argument by comparing $\left|p_{c}\left(R e^{i t}\right)-R^{n} e^{i n t}\right|$ with $\left|R^{n} e^{i n t}\right|$ for sufficiently large $R$.

We next demonstrate that the order of each zero is either 1 or -1 . Consequently, counting the number of distinct zeros of $p_{c}(z)$ is equivalent to counting the number of zeros according to multiplicity.

Lemma 2.2. All zeros of $p_{c}(z)=z^{n}+c \bar{z}^{k}-1$ have order 1 or -1 .

Proof. The trinomial $p_{c}(z)$ can be written as $p_{c}(z)=h+\bar{g}=\left(z^{n}-1\right)+\overline{\left(c z^{k}\right)}$. Let $z_{0}$ be a zero of $p_{c}(z)$. We know that $z_{0} \neq 0$ since $p_{c}(0)=-1$. The series expansions of $h$ and $g$ about $z_{0}$ are finite series since $h$ and $g$ are polynomials, where in accordance with the notation of equation (1.1),$a_{1}=n z_{0}^{n-1}$ and $b_{1}=c k z_{0}^{k-1}$, neither of which is zero. Thus the order of the zero at $z_{0}$ is either +1 if $\left|z_{0}\right|>R_{c}$ or -1 if $\left|z_{0}\right|<R_{c}$.

As already noted, the image of the critical circle $p_{c}\left(\Gamma_{c}\right)$ plays an important role in our analysis. We show that $p_{c}\left(\Gamma_{c}\right)$ is a hypocycloid of type $(n+k, n)$ per the following definition.

Definition 2.3 ([10]). A hypocycloid centered at the origin is the curve traced by a fixed point on a circle of radius $b$ rolling inside a larger origin-centered circle of radius $a$. The curve is given by the parametric equations

$$
\begin{aligned}
& x(\phi)=(a-b) \cos (\phi)+b \cos \left(\frac{a-b}{b} \phi\right), \\
& y(\phi)=(a-b) \sin (\phi)-b \sin \left(\frac{a-b}{b} \phi\right) .
\end{aligned}
$$

If the ratio $\frac{a}{b}$ is written in reduced form as $\frac{p}{q} \in \mathbb{Q}$, then the hypocycloid has $p$ cusps, and each arc connects cusps that are $q$ away from each other in a counterclockwise direction. Such a hypocycloid is called a $(p, q)$ hypocycloid, and the range of $\phi$ values to trace the entire hypocycloid is $0 \leq \phi \leq 2 \pi q$.

Lemma 2.4. The image $p_{c}\left(\Gamma_{c}\right)$ of the critical circle is an $(n+k, n)$ hypocycloid centered at $z=-1$. The value of $c$ affects only the size of the hypocycloid.

Proof. Evaluating $p_{c}\left(R_{c} e^{i \theta}\right)=R_{c}^{n} e^{i n \theta}+c R_{c}^{k} e^{-i k \theta}-1$ and splitting into real and imaginary parts gives

$$
u\left(R_{c} e^{i \theta}\right)=c^{\frac{n}{n-k}}\left[\left(\frac{k}{n}\right)^{\frac{n}{n-k}} \cos n \theta+\left(\frac{k}{n}\right)^{\frac{k}{n-k}} \cos k \theta\right]-1
$$


and

$$
v\left(R_{c} e^{i \theta}\right)=c^{\frac{n}{n-k}}\left[\left(\frac{k}{n}\right)^{\frac{n}{n-k}} \sin n \theta-\left(\frac{k}{n}\right)^{\frac{k}{n-k}} \sin k \theta\right] .
$$

We make the substitutions $b=c^{\frac{n}{n-k}}\left(\frac{k}{n}\right)^{\frac{k}{n-k}}, a=c^{\frac{n}{n-k}}\left(\frac{k}{n}\right)^{\frac{k}{n-k}}\left(\frac{n+k}{n}\right)$, and $n \theta=\phi$. As a consequence, we have $a-b=c^{\frac{n}{n-k}}\left(\frac{k}{n}\right)^{\frac{n}{n-k}}, \frac{a-b}{b}=\frac{k}{n}$, and $k \theta=\frac{a-b}{b} \phi$. Comparing with Definition 2.3, we see that the equations for $u$ and $v$ describe a hypocycloid centered at $z=-1$ instead of the origin. We observe that the ratio $\frac{a}{b}=\frac{n+k}{n}$ does not depend upon the constant $c$, and the entire hypocycloid is traced for $0 \leq \theta \leq 2 \pi$.

Because $b / a>1 / 2$, the smaller circle has more than half the radius of the larger circle. Thus, although the inner circle travels in a counterclockwise direction, the hypocycloid traces around its center in a clockwise direction.

It follows from Lemma 2.4 that in Example 1.2 with $p_{c}(z)=z^{5}+c \bar{z}^{3}-1, p_{c}\left(\Gamma_{c}\right)$ is an $(8,5)$ hypocycloid. Figure 2 exhibits this hypocycloid for three different values of $c$.

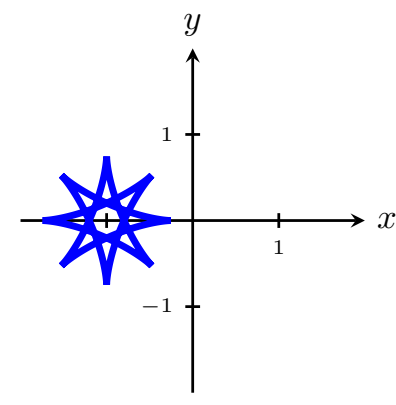

(A) $p_{1}\left(\Gamma_{1}\right)$

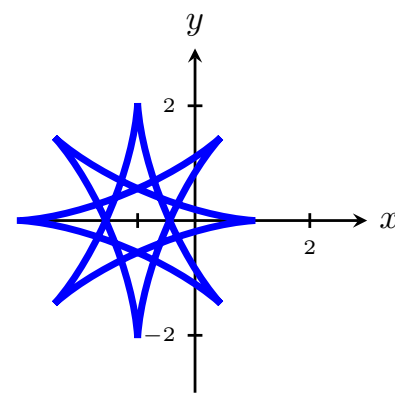

(B) $p_{1.5}\left(\Gamma_{1.5}\right)$

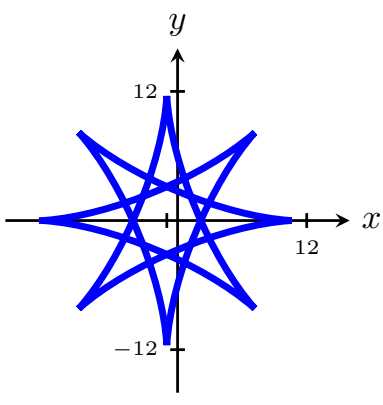

(C) $p_{3}\left(\Gamma_{3}\right)$

Figure 2. The $(8,5)$ hypocycloids $p_{c}\left(\Gamma_{c}\right)$ for $c=1,1.5$, and 3 .

Note that any two cusps that are $q$ cusps apart in the counterclockwise direction can also be viewed as being $p-q$ cusps apart in the clockwise direction. For the $(8,5)$ hypocycloids of Figure 2 each cusp is connected by an arc to cusps that are 3 cusps away, one in the counterclockwise and one in the clockwise direction.

In the following argument we make use of the geometry of the hypocycloid. For any value of $c$, the number of zeros of $p_{c}(z)$ in the sense-reversing region of the plane can be determined by finding the winding number $W_{c}$ of the hypocycloid $p_{c}\left(\Gamma_{c}\right)$ about the origin. For $c$ sufficiently small, the hypocycloid is entirely to the left of the origin, so $W_{c}=0$. As $c$ increases, $W_{c}$ remains constant until the hypocycloid passes through the origin, which occurs for the first time when $c$ equals the first critical value $c_{1}$. As $c$ continues to increase, there will be other critical values $c_{1}<c_{2}<\ldots<c_{N}$ for which the hypocycloid passes through the origin. These are the only places where the winding number $W_{c}$ can change. Counting the number of critical values thus requires us to first count the number of intersections of an $(n+k, n)$ hypocycloid with the portion of the real axis to the right of its center.

Lemma 2.5. The hypocycloid $p_{c}\left(\Gamma_{c}\right)$ has $N=\lfloor k / 2\rfloor+1$ distinct intersections with the real axis to the right of its center. These intersections, in turn, correspond to 
the $N$ critical c-values $c_{j}$, with $0<c_{1}<\ldots<c_{N}$, at which $p_{c}\left(\Gamma_{c}\right)$ intersects the origin. The winding number, $W_{c}$, of $p_{c}\left(\Gamma_{c}\right)$ around the origin is as follows:

(a) If $0<c<c_{1}$, then $W_{c}=0$.

(b) If $c_{j}<c<c_{j+1}$ for $1 \leq j \leq N-1$, then $W_{c}=-2 j+1$.

(c) If $c>c_{N}$, then $W_{c}=-k$.

Proof. We first show that the number of distinct intersections of the hypocycloid with the real axis to the right of its center is $N=\lfloor k / 2\rfloor+1$. The rightmost cusp of the hypocycloid corresponds to $\theta=0$ in the parameterization $p_{c}\left(R_{c} e^{i \theta}\right)$. Hence, solving the equation $p_{c}\left(R_{c}\right)=0$ for $c$ gives the first transition value. We easily obtain $c_{1}=\left(\frac{n}{n+k}\right)\left(\frac{n+k}{k}\right)^{k / n}>1$. Thus for $0 \leq c<c_{1}$, the hypocycloid lies entirely to the left of the origin.

We label the rightmost cusp of the hypocycloid with 0 , and the other cusps in a counterclockwise direction with $1,2, \ldots, n+k-1$. Note that labels on cusps must be computed modulo $n+k$. For example, the cusp with label $n+k-r$ can also be referred to using the label $-r$. Two arcs emanate from each cusp, and each arc connects cusps that are $k$ apart. Since $k<(n+k) / 2$, cusp $k$ is in the upper half plane, and connects to cusp 0 on the real axis. Based on the labeling of cusps and the geometry of the hypocycloid, each cusp labeled $1,2, \ldots k-1$ has an arc that connects to a cusp in the lower half plane and crosses the real axis to the right of the center of the hypocycloid. No other arcs can cross this portion of the real axis. When combined with the arc from cusp $k$ to cusp 0 , we see that each cusp labeled $1,2, \ldots, k$ is the end point of one arc that intersects the real axis to the right of center.

The hypocycloid is symmetric with respect to the real axis, however, so not all $k$ intersections with the real axis are unique. Let $\gamma\left(r, r^{\prime}\right)$ denote the arc of the hypocycloid that connects cusps $r$ and $r^{\prime}$. Then for each cusp $r$ with $1 \leq r \leq k-1$, the $\operatorname{arcs} \gamma(r, r-k)$ and $\gamma(-r,-r+k)$ pass through the same point on the real axis. These two arcs are distinct except in the case where $r-k=-r \bmod (n+k)$. This modular equation is equivalent to $2 r=k \bmod (n+k)$, which is only satisfied when $k$ is even and $r=k / 2$. We also observe that an arc which is its own mirror image must be the leftmost intersection of the hypocycloid with the real axis that lies to the right of the center.

Thus, for $k$ odd, every distinct intersection involves a pair of arcs, except for the arc $\gamma(0, k)$. So in this case there are $1+(k-1) / 2$ distinct intersections. For the case $k$ even, there are $1+(k-2) / 2+1$ distinct intersections. These formulations can be combined using the floor function to give $N=\lfloor k / 2\rfloor+1$ distinct intersections for all values of $k$.

Now we compute the winding number $W_{c}$, using the crossing condition as stated in [15]. By that condition, we only need to consider a path from the origin going to the right, and count how many curves we must cross to "escape" the hypocycloid. Each curve that we cross that is moving from our right to left as we travel away from the origin contributes -1 to $W_{c}$. Combining the crossing condition with the discussion above, we see that as $c$ increases, the winding number $W_{c}$ changes according to the pattern $0,-1,-3,-5, \ldots$, which is expressed in part (b). Once $c$ is large enough that all intersections with the real axis to the right of the center of the hypocycloid are also to the right of the origin, we have $W_{c}=-k$. Note that in 
the case $n$ odd and $k$ even, the last change in the winding number is from $-k-1$ to $-k$.

Each change in the winding number occurs at a critical value of $c_{j}$. Thus there are $N=\lfloor k / 2\rfloor+1$ such values.

We are now prepared to prove the main theorem.

Proof of Theorem 1.1. By Lemma 2.1 and the Argument Principle for Harmonic Functions, the sum of the orders of the zeros of $p_{c}(z)$ is always $n$. Furthermore, by Lemma 2.2 all zeros of $p_{c}(z)$ have order 1 or -1 . Since $p_{c}\left(\Gamma_{c}\right)$ is completely to the left of zero when $0<c<c_{1}$, we conclude that for such $c, p_{c}(z)$ has only $n$ zeros. We next discuss $c_{1}<c<c_{2}$. Since $W_{c}=-1$, there is one zero inside $\Gamma_{c}$, so there must be $n+1$ zeros outside of $\Gamma_{c}$, for a total of $n+2$ zeros. Now, when $c_{j}<c<c_{j+1}$ for $2 \leq j \leq N-1$, we have $W_{c}=1-2 j$. We apply the Argument Principle to say that the number of zeros in the sense-preserving region of the plane must be $n+|1-2 j|=n+2 j-1$. Thus the total number of zeros is $n+2(2 j-1)=n+4 j-2$, as desired. Similarly, since $W_{c}=-k$ for $c>c_{N}$, we have that $p_{c}(z)$ has $n+2 k$ zeros in this case.

Finally, we note that the upper and lower bounds for $c_{1}$ and $c_{N}$ can be found directly using the triangle inequality by examining for what $c$ values $p_{c}\left(\Gamma_{c}\right)$ goes through the origin. The condition that $p_{c}\left(R_{c} e^{i \theta}\right)=0$ implies that

$$
c^{\frac{n}{n-k}}\left|\left(\frac{k}{n}\right)^{\frac{n}{n-k}} e^{i(k+n) \theta}+\left(\frac{k}{n}\right)^{\frac{k}{n-k}}\right|=1 .
$$

Applying the triangle inequality, we have that

$$
\left(\frac{k}{n}\right)^{\frac{k}{n-k}}-\left(\frac{k}{n}\right)^{\frac{n}{n-k}} \leq\left|\left(\frac{k}{n}\right)^{\frac{n}{n-k}} e^{i(k+n) \theta}+\left(\frac{k}{n}\right)^{\frac{k}{n-k}}\right| \leq\left(\frac{k}{n}\right)^{\frac{n}{n-k}}+\left(\frac{k}{n}\right)^{\frac{k}{n-k}}
$$

or, equivalently, that

$$
\left(\frac{k}{n}\right)^{\frac{k}{n-k}}\left(\frac{n-k}{n}\right) \leq\left|\left(\frac{k}{n}\right)^{\frac{n}{n-k}} e^{i(k+n) \theta}+\left(\frac{k}{n}\right)^{\frac{k}{n-k}}\right| \leq\left(\frac{k}{n}\right)^{\frac{k}{n-k}}\left(\frac{n+k}{n}\right) .
$$

This, in turn, implies that

$$
\frac{n}{n+k}\left(\frac{n+k}{k}\right)^{\frac{k}{n}} \leq c \leq \frac{n}{n-k}\left(\frac{n-k}{k}\right)^{\frac{k}{n}} .
$$

Since $1<c_{1}<c_{N}<2$, all the transitions from $n$ zeros to $n+2 k$ zeros occurs within a small range of real numbers.

\section{Areas FOR FURTHER INVESTigation}

(1) In this paper, we showed how the number of zeros changes as $c$ varies for $p_{c}(z)=z^{n}+c \bar{z}^{k}-1$, but we did not prove anything about the location of the zeros. What can be shown about the location of the zeros for $p_{c}(z)$ ?

(2) What are the exact values for $c_{j}$ for $1<j<N$ ?

(3) The subclass of harmonic polynomials $p(z)=h(z)-\bar{z}$ is related to gravitational lensing, and it has been shown [9] that if the degree of $h$ is $n>1$, then the number of zeros is bounded by $3 n-2$. Could the approach of this paper be used to improve that bound or to show that it is sharp? 
(4) Our proof of Theorem 1.1 relies heavily on the fact that the image of the critical circle is a hypocycloid with a cusp on the real axis. If $c$ is complex but not real, the image curve no longer has this simple geometry. What is the analogue of Theorem 1.1 for complex $c$ ?

(5) What can be proven about the number of zeros of other families of harmonic polynomials?

\section{REFERENCES}

[1] Michael A. Brilleslyper and Lisbeth E. Schaubroeck, Locating unimodular roots, College Math. J. 45 (2014), no. 3, 162-168, DOI 10.4169/college.math.j.45.3.162. MR3207562

[2] Michael A. Brilleslyper and Lisbeth E. Schaubroeck, Counting interior roots of trinomials, Math. Mag. 91 (2018), no. 2, 142-150, DOI 10.1080/0025570X.2017.1420332. MR.3777918

[3] Daoud Bshouty, Walter Hengartner, and Tiferet Suez, The exact bound on the number of zeros of harmonic polynomials, J. Anal. Math. 67 (1995), 207-218, DOI 10.1007/BF02787790. MR 1383494

[4] J. Clunie and T. Sheil-Small, Harmonic univalent functions, Ann. Acad. Sci. Fenn. Ser. A I Math. 9 (1984), 3-25, DOI 10.5186/aasfm.1984.0905. MR752388

[5] Michael J. Dorff and James S. Rolf, Anamorphosis, mapping problems, and harmonic univalent functions, Explorations in complex analysis, Classr. Res. Mater. Ser., Math. Assoc. America, Washington, DC, 2012, pp. 197-269. MR2963968

[6] Peter Duren, Harmonic mappings in the plane, Cambridge Tracts in Mathematics, vol. 156, Cambridge University Press, Cambridge, 2004. MR2048384

[7] Peter Duren, Walter Hengartner, and Richard S. Laugesen, The argument principle for harmonic functions, Amer. Math. Monthly 103 (1996), no. 5, 411-415, DOI 10.2307/2974933. MR 1400723

[8] Russell Howell and David Kyle, Locating trinomial zeros, Involve 11 (2018), no. 4, 711-720, DOI 10.2140/involve.2018.11.711. MR3778921

[9] Dmitry Khavinson and Grzegorz Świątek, On the number of zeros of certain harmonic polynomials, Proc. Amer. Math. Soc. 131 (2003), no. 2, 409-414, DOI 10.1090/S0002-9939-0206476-6. MR.1933331

[10] Marie M. Johnson, Book Review: Analytic Geometry by Charles H. Lehmann, Natl. Math. Mag. 17 (1943), no. 6, 280. MR.1570119

[11] O. Lehto and K. I. Virtanen, Quasiconformal mappings in the plane, 2nd ed., Springer-Verlag, New York-Heidelberg, 1973. Translated from the German by K. W. Lucas; Die Grundlehren der mathematischen Wissenschaften, Band 126. MR0344463

[12] Seung-Yeop Lee, Antonio Lerario, and Erik Lundberg, Remarks on Wilmshurst's theorem, Indiana Univ. Math. J. 64 (2015), no. 4, 1153-1167, DOI 10.1512/iumj.2015.64.5526. MR.3385788

[13] Hans Lewy, On the non-vanishing of the Jacobian in certain one-to-one mappings, Bull. Amer. Math. Soc. 42 (1936), no. 10, 689-692, DOI 10.1090/S0002-9904-1936-06397-4. MR.1563404

[14] Aaron Melman, Geometry of trinomials, Pacific J. Math. 259 (2012), no. 1, 141-159, DOI 10.2140/pjm.2012.259.141. MR 2988487

[15] Tristan Needham, Visual complex analysis, The Clarendon Press, Oxford University Press, New York, 1997. MR 1446490

[16] R. Peretz and J. Schmid, Proceedings of the Ashkelon Workshop on Complex Function Theory, (1996), 203-208, Bar-Ilan Univ., Ramat Gan, 1997.

[17] T. Sheil-Small in Tagesbericht, Mathematisches Forsch. Inst. Oberwolfach, Funktionentheorie, 16-22.2.1992, 19.

[18] A. S. Wilmshurst, The valence of harmonic polynomials, Proc. Amer. Math. Soc. 126 (1998), no. 7, 2077-2081, DOI 10.1090/S0002-9939-98-04315-9. MR.1443416 
Department of Mathematical Sciences, United States Air Force Academy, USAF ACADEmy, Colorado 80840

Email address: mike.brilleslyper@usafa.edu

Department of Mathematics, Brigham Young University, Provo, Utah 84602

Email address: jbrooks@mathematics.byu.edu

Department of Mathematics, Brigham Young University, Provo, Utah 84602

Email address: mdorff@math. byu.edu

Department of Mathematics and Computer Science, Westmont College, Santa BarBARA, CALifornia 93108

Email address: howell@westmont.edu

Department of Mathematical Sciences, United States Air Force Academy, USAF ACAdemy, Colorado 80840

Email address: beth.schaubroeck@usafa.edu 